\title{
OPTIMAL PRESTRESS AGAINST BUCKLING: AN ENERGY APPROACH $\dagger$
}

\author{
J. E. TAYIOR: \\ The University of Michigan, Ann Arbor
}

\begin{abstract}
Structures that have a reserve capacity beyond buckling can generally be stiffened against buckling through the use of prestress. The design of prestress for such cases is treated in a way which leads to the maximization of the buckling load for a prescribed available prestress strain encrgy. The formulation given for this optimal design problem relates closely to the energy principles of structural analysis. Optimal prestress configurations are determined for the circular plate, and for a second example structure in the form of a stut-membrane system. Developments from the study of prestress design are related to past results from postbuckling analysis.
\end{abstract}

\section{INTRODUCTION}

THIs paper treats a category of problems among those where the response of a structure depends in a substantial way on initial stress state. Specifically, the object of the study is to determine how to best predispose a structure via prestress in such a way as to defer buckling. The structural form itself is taken to be fixed.

The idea of prestressing this way is sensible only for structures that exhibit redundancy relative to buckling. Consider for example a statically redundant truss, loaded to the point where the member most susceptible to buckling reaches its critical state. Buckling of the member is reflected in the truss as a softening rather than collapse. It is generally possible in this situation to pretension the particular member via prestress. This has the effect of raising the external load at which it buckles, thereby stiffening the truss.

Plates can be prestressed against buckling as well. Stoker [1] investigated certain prestress configurations for stiffening the circular plate. Although the mechanism by which the plate becomes stiffened is obscure relative to the truss example, the situation is in fact much the same. Redundancy rclative to buckling is identified in plates with their capacity to sustain loads beyond the critical value. The (first mode) buckling load may be increased via a prestress field that pretensions the interior or least well supported regions of the plate.

As noted, it is possible to stiffen a structure by prestress only where some characteristic redundancy is present. On the other hand, it seems to hold in general that where the redundancy exists, the occurrence of "first buckling" can be deferred. Prestress might be used to advantage in the design of stay-support masts, rib-membrane structures, and beams relative to lateral buckling, to name but a few other examples. However, the merit of using prestress in this way depends a great deal on the type of structure, the nature of its use, relative

† This study received support from the National Science Foundation under grant GK 11356.

‡ Associate Professor, Departments of Aerospace Engineering and Engineering Mechanics. 
costs, and so forth. It should also be noted that the ultimate capacity of a structure is in general unaffected by the type of prestress considered in this study. (This is implicit in the results of several studies on the collapse after buckling of redundant structure, e. Masur [2], Masur and Milbradt [3].) Therefore, wherever design is more sensibly based on ultimate capacity, the subject prestress is of no use.

Given that there is purpose in prestressing against buckling, the objective of this study is to provide a theory for the prediction of an optimal prestress configuration. The optimal prestress is simply the one which maximizes the "first-buckling" load. The optimization problem is formulated in a way that relates closely to the conventional energy principles of structural analysis. This representation of the prestress design problem parallels the developments in [4] and [5] of energy formulations for the optimal design of member shape. As in those cases, the analytics takes the form of an isoperimetric problem in the calculus of variations.

The theory is demonstrated for two structural forms, the plate, and a bar-membrane model. Numerical results provide some measure of the effectiveness of prestress for these forms. A proof of optimality (written for the plate) substantiates the identity between extremization of energy and maximization of the buckling load.

\section{OPTIMUM PRESTRESS OF PLATES}

As noted in the introduction, the optimum prestress problem is treated here in a form which relates to the common variational methods of analysis. Also, the present purposes are served by nothing deeper among plate theories than the classical Kirchoff plate model. In other words, the governing equations for the prestress design problem are derived for the classical model via an energy formulation. The equations are solved for the example of a simply supported uniform circular plate.

For plate buckling analysis, the change in potential energy associated with the buckling process may be expressed as (for example, see [6]):

$$
G=\frac{1}{2} \int_{R} N_{i j} w_{r_{i}} w_{{ }_{j}} \mathrm{~d} A+\frac{D}{2} \int_{R}\left[(1-v) w_{r_{i j}} w_{i j}+v w_{{ }^{\prime} k} w_{\cdot_{q q}}\right] \mathrm{d} A
$$

$w\left(x_{i}\right)$ symbolizes lateral displacement of the plate from the fiat to the buckled configuration, while $N_{i j}$ represents the total in-plane stress resultant at buckling. The condition $\delta G=0$ leads to the familiar equation

$$
D w_{i i j j}=N_{i j} w_{i} w,_{j}
$$

and associated boundary and corner conditions.

A prestress field, say $N_{i j}^{o}$, is defined by

$$
\begin{array}{cc}
N_{i j^{\prime} j}^{0}=0 & \text { over the plate region } R \\
N_{i j}^{0} n_{j}=0 & \text { on the plate boundary } B
\end{array}
$$

The vector $n_{j}$ is a unit outer normal on $B$. Where such a self-equilibrated stress field occurs, the total in-plane stresses $N_{i, j}$ include the $N_{i j}^{0}$ and the change in the field, say $P \bar{N}_{i j}$, associated with the edge loading, i.e.

$$
N_{i j}=N_{i j}^{0}-P \bar{N}_{i j}
$$


The $\bar{N}_{i j}$ satisfy

$$
\begin{gathered}
\bar{N}_{i j^{\prime} j}=0 \quad \text { in } R \\
\bar{N}_{i j} n_{j}=-\tau_{i} \text { on B }
\end{gathered}
$$

$P \tau_{i}$ represents the specified edge tractions. The constant $P$ measures magnitude of the load; $P>0$ for compression. Equations $(2)(5)$ summarize the analysis problem for the plate with prestress $N_{i j}^{0}$.

The present treatment for optimum prestress may be described through the problem statement:

Determine from among all admissible fields $N_{i j}^{0}$ the one which maximizes the buckling load $P$. The admissible $N_{i j}^{0}$ includes all self-equilibrated fields of specified total strain energy.

The relevance in a technical sense of such an approach for the design of prestress is discussed in Section 5. The solution of this problem is identified with an extremum within the stated constraint on prestress energy of the energy $G$. This identity is verified in Section 4 .

In order to state concisely the energy $U_{0}=\frac{1}{2} \int_{R} N_{i j}^{0} e_{i j}^{0} \mathrm{~d} A$ of self equilibrated stress fields, the stress function $\phi$ is introduced:

$$
N_{i j}^{0}=\phi \cdot_{k k} \delta_{i j}-\phi^{*}{ }_{i j}
$$

The simple material law

$$
e_{i j}^{0}=a N_{i j}^{0}+b N_{k k}^{0} \delta_{i j}
$$

( $a$ and $b$ are the usual material constants for an isotropic material) is applied along with (6) to obtain

$$
U_{0}=\frac{1}{2} \int_{R}\left(a \phi_{\cdot_{i j}} \phi_{\cdot_{i j}}+b \phi_{{ }^{\prime} k} \phi_{{ }^{\prime} q}\right) \mathrm{d} A
$$

For specified magnitude of prestress energy $C$, the condition

$$
U_{0}-C=0
$$

summarizes admissibility. Recall that the $\phi$ are required to meet boundary condition (3).

The constraint on $U_{0}$ is appended to cnergy $G$ in the usual way:

$$
\begin{aligned}
H= & G-\lambda\left(U_{0}-C\right) \\
= & \int_{R}\left\{\frac{D}{2}\left[(1-v) w_{i_{i j} w_{i j}}+v w_{r_{k}} w_{r_{q q}}\right]+\frac{1}{2}\left[-P \bar{N}_{i j}+\left(\phi_{\cdot_{k k}} \delta_{i j}-\phi_{\cdot_{i j}}\right)\right] w_{r_{i}} w_{\cdot j}\right\} \mathrm{d} A \\
& -\lambda\left[\frac{1}{2} \int_{R}\left(a \phi_{\prime_{i j}} \phi_{\cdot_{i j}}+b \phi_{\cdot_{k k}} \phi \cdot_{q q}\right) \mathrm{d} A-C\right]
\end{aligned}
$$

The solution of the prestress design problem turns out to be an extremal of the constrained functional $H$. Thus the governing equations are equation (2), rewritten here in terms of $\phi$, $P$ and $\bar{N}_{i j}$,

$$
D w_{i i j j}=\left(-P \bar{N}_{i j}+\phi_{r_{k k}} \delta_{i j}-\phi_{r_{i j}}\right) w_{i j}
$$


with the associated boundary conditions, and

$$
\lambda(a+b) \phi \dot{\phi}_{i i j j}-\frac{1}{2}\left(w_{\cdot i j} w_{i, j}-w_{\cdot i} w_{\cdot j j}\right)=0
$$

In other words, the functions $\phi$ and $w$ which satisfy equations (10) and (11) represent the optimum prestress and associated buckling response. Once again, this is to be verified in Section 4.

\section{Solution for the circular plate}

The solution is to be developed for the simply supported circular plate under uniform edge thrust $p$, within the assumption that the buckled shape is axisymmetric. For this case equations (10) and (11) may be reduced to

$$
\begin{aligned}
& \frac{\mathrm{d}}{\mathrm{d} r}\left[\frac{D}{r} \frac{\mathrm{d}}{\mathrm{d} r}(r \beta)\right]=\left(N^{0}-p\right) \beta \\
& \frac{1}{r \mathrm{~d} r}\left(r^{3} \frac{\mathrm{d} N^{0}}{\mathrm{~d} r}\right)=\frac{1}{2 \lambda(a+b)} \beta^{2}
\end{aligned}
$$

in terms of:

$$
\begin{aligned}
\beta & =\mathrm{d} w / \mathrm{d} r \\
N^{\circ} & =\text { the radial component of the stress field }
\end{aligned}
$$

The equations are now stated more simply in terms of the resultant $N^{0}$, rather than the stress function. At the edge $r=\bar{r}$,

$$
\begin{aligned}
& \left.\frac{\mathrm{d} \beta}{\mathrm{d} r}\right|_{r=r}=0 \\
& \left.N^{0}\right|_{r=r}=0
\end{aligned}
$$

Also,

$$
\left.\beta\right|_{r=0}=0
$$

To facilitate the development of a numerical solution, the system $(12)-(16)$ is transformed as follows

$$
\begin{aligned}
0 \leqq \rho=r / \bar{r} \leqq 1 ; & \pi=p \bar{r}^{2} / D \\
\eta=N^{0} \bar{r}^{2} / D ; & \eta=\bar{r} \beta /[2 \lambda(a+b) D]^{\frac{1}{2}}
\end{aligned}
$$

In the nondimensional form,

$$
\begin{aligned}
{\left[(\rho \gamma)^{\prime} / \rho\right]^{\prime} } & =(\eta-\pi) \gamma \\
\left(\rho^{3} \eta^{\prime}\right)^{\prime} / \rho & =-\eta^{2} \\
\eta^{\prime}(1) & =0 \\
\eta(1) & =0 \\
\gamma(0) & =0
\end{aligned}
$$

Prime indicates derivative with respect to $\rho$. 
Solutions of the system (18) are obtained for a set of values of the load parameter $\pi$. (Numerical results were generated via a simple iterative scheme; Friedrichs and Stoker [7] used alternatively perturbations and power series expansions to treat the same equations.) Typical prestress fields are shown in Fig. 1. Figure 2 shows the effect on buckled shape of increasing levels of prestress. In this problem, the assumptions of axisymmetry and continued elastic response allow that the model predicts $\pi \rightarrow \infty$ as the prestress energy increases without bound. The corresponding situation for the postbuckled plate is described in [7].

\section{A BAR-MEMBRANE MODEL}

As a second application of the theory, the prestress is to be designed for a structure comprised of a membrane attached to a bar or rib (See Fig. 3). The membrane and bar are viewed as components which may be specified individually, so the structure is a two

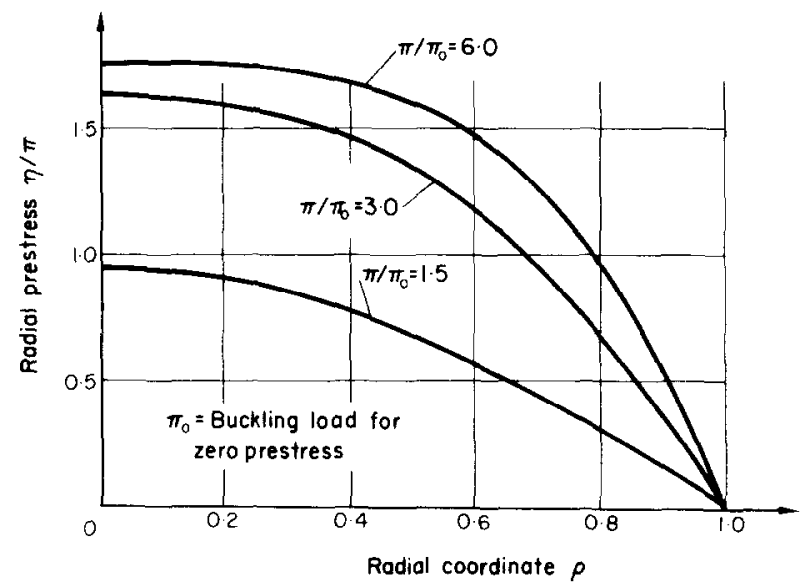

Fig. 1. Typical radial prestress fields for the simply supported circular plate.

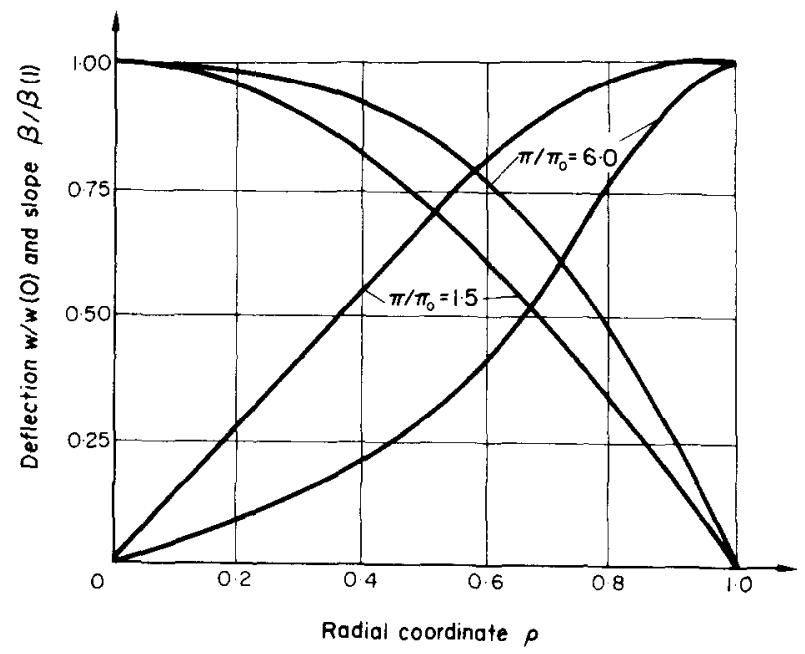

FIG. 2. Typical buckling modes for the prestressed circular plate. 

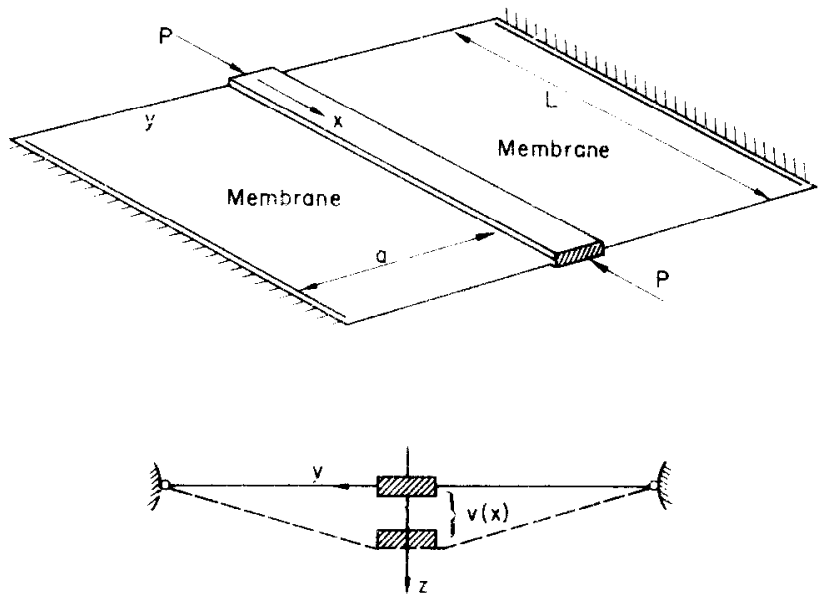

Fic: 3. The bar-membrane siructural system.

element system. Since the prestress is associated with the membrane only, certain features of the prestress design problems are revealed more clearly through the treatment of this mode! than in the study of the (single-element) plate.

The bar-membrane model may be thought to simulate a portion of a pressurestabilized rib-reinforced structure. Thus it is of some technical interest to recognize what can be accomplished here through prestress design. On the other hand, the analytics for this model involves nothing new over the development for the plate. For simplicity, the membrane stress field is taken to be one dimensional, i.e. suppose the membrane is attached in a way that admits the stress resultant field $N_{x x}^{0}=N_{x y}^{0} \equiv 0 ; N_{y y}^{0}=N^{0}(x)$. The governing functional is then [much like $H$ of $(9)]$ :

$$
2 H=\int_{0}^{L}\left(\frac{N^{0} v^{2}}{a}+E I v^{\prime \prime 2}-P v^{\prime 2}\right) \mathrm{d} x-\lambda\left(\frac{1}{k} \int_{0}^{L} N^{02} \mathrm{~d} x-2 C\right)
$$

expressed in terms of

$$
\begin{aligned}
E I & =\text { flexural rigidity of the bar } \\
k & =\text { stiffness of the membrane } \\
a & =\text { length of the membrane } \\
L & =\text { length of the bar } \\
v(x) & =\text { lateral displacement of the bar } \\
\lambda & =\text { constant multiplier. }
\end{aligned}
$$

Prime indicates derivative with respect to $x$. The first term in (19) reflects the bar-membrane interaction. Constant $C$ again represents the specified magnitude of prestress energy. The coordinates, dimensions, and so forth are indicated in Fig. 3. The possibility of buckling in the plane of the membrane is excluded.

The governing equations $\delta H=0$ are, from (19)

$$
\left(E I v^{\prime \prime}\right)^{\prime \prime}+P v^{\prime \prime}+N^{0} v / a=0
$$


with associated boundary conditions and

$$
v^{2}-2 a \lambda N^{0} / k=0
$$

The solution of $(20),(21)$, say $\left(N^{0 *} ; v^{*} ; P^{*}\right)$, represents the optimum prestress and associated buckling load and configuration. Again, the optimal character of this solution is demonstrated in Section 4.

\section{Solutions}

The system (20), (21) is put into nondimensional form by the transformation:

$$
-1 \leqq\left(z=\frac{2 x}{L}-1\right) \leqq 1 ; \quad u=\alpha v ; \quad \bar{N}=n N^{0}
$$

in which the constants $\alpha$ and $n$ are

$$
\alpha=\frac{L}{2 a} \sqrt{\left(\frac{k}{2 P \lambda}\right)} \quad n=\frac{L^{2}}{A P a}
$$

In terms of $u$ and $\bar{N}$

$$
\begin{gathered}
\kappa u^{\prime \prime \prime}+u^{\prime \prime}+u \bar{N}=0 \\
u^{2}-\bar{N}=0
\end{gathered}
$$

or if $\bar{N}$ is eliminated,

$$
\kappa u^{\prime \prime \prime \prime}+u^{\prime \prime}+u^{3}=0
$$

The parameter $\kappa$ is

$$
\kappa=4 E I / P L^{2}
$$

or with the end load $P$ expressed as $P=r^{2} E I / L^{2}$,

$$
\kappa=4 / r^{2}
$$

For membrane stiffness $k>0$, the buckling load $r^{2}>r_{1}^{2}=$ eigenvalue for the unsupported bar. Thus the range of $\kappa$ is $0 \leqq \kappa \leqq 4 / r_{1}^{2}$. Solutions are obtained via an iterative scheme based on the equation

$$
\kappa u_{k}^{\prime \prime \prime \prime}+u_{k}^{\prime \prime}=-u_{k-1}^{3}
$$

Typical results for the simply supported bar are given in Fig. 4.

For the limit value $\kappa=0$, equation (25) may be integrated directly. The solution in terms of elliptic functions is

$$
F\left[\cos ^{-1} \frac{w}{\sqrt{C}} ; \frac{1}{\sqrt{ } 2}\right]=(2 \sqrt{ } C) z
$$

In this case of zero flexural rigidity, one might take the bar to be comprised of a set of rigid blocks hinged together. Thus the structural capacity depends wholly on the membrane prestress. The optimum system is compared for this case to one for which the membrane has uniform prestress. With equal prestress energies, the ratio of buckling loads turns out to be $5 / 4$. 


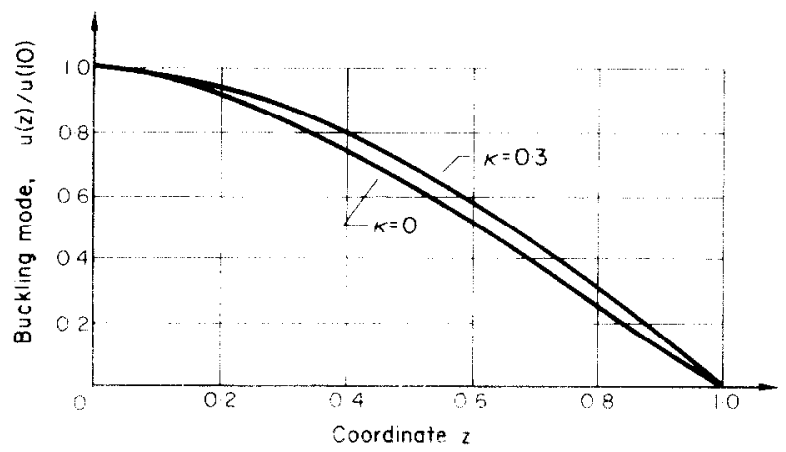

Fig. 4. Typical buckling modes for the bar membrane structure.

\section{PROOF OF OPTIMALITY}

The object is to show that the necessary conditions for the characteristic energy functional $H$ to be stationary are in fact necessary and sufficient for the optimization problem stated in Section 2. In other words, it is to be proved that the solution of equations (10), (11) or (20), (21) correspond to maximization of the buckling loads in the respective cases. The proof is written specifically for the plate problem.

For convenience in writing the proof. the $w\left(x_{i}\right)$ are taken to be normalized such that

$$
\frac{1}{2} \int_{R} \bar{N}_{i j} w_{i} w_{i j} \mathrm{~d} A=1
$$

This in effect fixes the value of the unspecified multiplier $\lambda$. The value of $\lambda$ is itself unimportant. Thus the potential energy is written

$$
G\left(w ; N_{i j}^{0}: P\right)=U\left(w ; N_{i j}^{0}\right)-P
$$

The form of strain energy $U$ follows the expression of energies in equation (9). For any admissible prestress field and the associated eigenfunction $w$ and eigenvalue $P$.

$$
G\left(w ; N_{i j}^{0} ; P\right)=0
$$

In particular, the equation holds for the solution, say $\left(w^{*} ; N_{i j}^{0 *} ; P^{*}\right)$. of equations (10). (11).

From equation (31) with the generic $\left(w ; N_{i j}^{0} ; P\right)$, and the same equation written for the starred solution, we have

$$
P^{*}-P=U\left(w^{*} ; N_{i j}^{0 *}\right)-U\left(w: N_{i j}^{0}\right)
$$

By the principle of minimum potential energy

$$
G\left(w ; N_{i j}^{0} ; P\right) \leqq G\left(w^{*} ; N_{i j}^{0} ; P\right)
$$

or in view of (30)

$$
U\left(w ; N_{i j}^{0}\right) \leqq U\left(w^{*} ; N_{i j}^{o}\right)
$$


Equations (32) and (33) lead to

$$
P^{*}-P \geqq U\left(w^{*} ; N_{i j}^{0 *}\right)-U\left(w^{*} ; N_{i j}^{0}\right)=\int_{R}\left(N_{i j}^{0 *}-N_{i j}^{0}\right) w_{i}^{*} w_{j}^{*} \mathrm{~d} A
$$

It may be established from (11) that,

$$
\int_{R} N_{i j}^{0}\left(w_{i}^{*} w_{j}^{*}-2 \lambda e_{i j}^{0 *}\right) \mathrm{d} A=0
$$

Equation (35) holds for any admissible $N_{i j}^{*}$, which of course includes the putative optimum field $N_{i j}^{0 *}$.

Making use of (35), (34) can be written

But

$$
P^{*}-P \geqq 2 \lambda \int_{R}\left(N_{i j}^{0 *}-N_{i j}^{0}\right) e_{i j}^{0 *} \mathrm{~d} A=2 \lambda\left(C-\int_{R} N_{i j}^{0} e_{i j}^{0 *} \mathrm{~d} A\right)
$$

$$
\frac{1}{2}\left(N_{i j}^{0 *}-N_{i j}^{0}\right)\left(e_{i j}^{0 *}-e_{i j}^{0}\right)=\frac{1}{2}\left(N_{i j}^{0 *} e_{i j}^{0 *}+N_{i j}^{0} e_{i j}^{0}-N_{i j}^{0 *} e_{i j}^{0}-N_{i j}^{0} e_{i j}^{0 *}\right) \geqq 0
$$

since the starred and unstarred fields are both actual solutions and therefore the product $\left(N_{i j}^{0 *}-N_{i j}^{0}\right)\left(e_{i j}^{0 *}-e_{i j}^{0}\right)$ is a measure of strain energy. Now $\frac{1}{2} N_{i j}^{0 *} e_{i j}^{0 *}=\frac{1}{2} N_{i j}^{0} e_{i j}^{0}=C$ by the admissibility condition on prestress fields, and $N_{i j}^{0 *} e_{i j}^{0}=N_{i j}^{0} e_{i j}^{0 *}$. Thus (37) furnishes

$$
2\left(C-\int_{R} N_{i j}^{0} e_{i j}^{0 *} \mathrm{~d} A\right) \geqq 0
$$

By comparison of (36) and (38), $P^{*}-P \geqq 0$ and the proof is complete.

The optimal prestress problem may now be summarized in several ways. It has been shown that:

1. The Euler-Lagrange equations (10), (11) for the functional $H$ are sufficient conditions for a maximum of the buckling load.

The solution of these equations is unique (aside from the sign of $w$ ) for loads $P$ no greater than the value at which buckling in a higher mode might occur. Thus the analytical characterization of the problem is complete. Recall that the admissibility conditions on prestress were incorporated in the formation of $H$.

In view of the result $P^{*}-P \geqq 0$ and equation (32), it follows as well that:

2. The admissible prestress field which maximizes the strain energy associated with buckling maximizes the buckling load.

A related, sharper statement on strain energies may be made:

3. The admissible prestress field that maximizes the part of the buckling strain energy associated with prestress maximizes the buckling load.

One approach to the proof of 3 follows closely the development in [6] of a minimum-energy principle for post-buckling behavior of plates.

Indeed, the post-buckling analysis and optimum design of prestress problems turn out to be closely related. The governing equations (10), (11) for plate prestress are precisely the same form as the familiar von Karman plate equations. This lends quantitative substance to the intuitively appealing idea that: for a given plate, the prestress field appropriate to 
maximization of the buckling load is that field which will have the total membrane stress at buckling equal to the post-buckling membrane stress for the initially stress free plate under the same load. Moreover, the buckling mode is the same as the post-buckling configuration of the unprestressed plate. The relation evidently holds for all structural forms where the prestress is coupled with quadratic terms in the buckling deformation.

Finally, it is noted without proof that the determination of the "minimum-energy prestress field" for specified buckling load is equivalent to the problem treated in this study. This corresponds to a common form of reciprocity among isoperimetric problems in the variational calculus $[8]$.

\section{SUMMARY}

Two applications of a theory for the design of prestress have been discussed in detail. The breadth of the theory itself may be judged for the most part from the problem description given in the Introduction. As noted there, the possibility of stiffening via prestress relates to the presence in the structure of a redundancy relative to buckling. This redundancy may in turn be identified quantitatively with the appropriate terms in a measure of the post-buckling strain energy of the unprestressed structure (see for example [3]). The form of proof of optimality given in Section 4 applies in general, as may be noted directly from the details of that development.

The prestress design problem has been formulated in a way that makes use of energy measures familiar in mechanics analysis. The same variational problem might be expressed easily enough in other forms. The equivalent minimum-maximum problem statement, for example, represents the conventional minimization relative to mode shape and a maximum relative to prestress field of the Rayleigh quotient for the eigenvalue. Minimization of prestress energy within a constraint reflecting the equations for buckling analysis provides the statement in the form of a Lagrange problem, and so on. It seems that among these various forms, the one used in this study leads most directly to a concise version of the complete boundary value problem statement. Of course this form also provides the convenience that one ordinarily associates with energy methods of analysis.

As noted in Section 2, the solution for the circular plate has the buckling load increase indefinitely with increasing prestress energy. Stoker [1] notes that this increase is in fact limited ; eventually the buckling mode ceases to be axisymmetric. In addition, if the assumptions of axisymmetry and continued elasticity are relaxed, the appropriate treatment of the problem would indicate the eventual development of either buckling or yielding as a result of the prestress itself. While some buckling under prestress (without yielding) might be tolerated, there is surely a limit level (ultimate capacity) for prestress. This identifies in one way an ultimate capacity of the prestressed plate. Buckling under prestress is not an issue with the bar-membrane structure, so in that case the limit on prestress relates to material capacity only.

The optimal prestress field was identified in Section 4 with the net postbuckled membrane stress of an initially stress-free plate. The condition for $H$ to be stationary relative to prestress resembles the derivation of a compatibility equation. But the second of the von Karman equations derives from the requirement that the membrane deformations must be compatible with the shape of the buckled plate. Thus the identity might have been recognized simply from the form of the governing functional $H$. 


\title{
REFERENCES
}

[1] J. J. Storer, Pre-stressing a Plane Circular Plate to Stiffen It Against Buckling, Reissner Anniv. Vol., p. 268. Edwards Bros. (1940).

[2] E. F. Masur, Lower and upper bounds to the ultimate loads of buckled redundant trusses. Q. appl. Math. 11, 385-392 (1954).

[3] E. F. Masur and K. P. Mir.radat, On the carrying capacity of redundant structures. J. appl. Mech. 23. 403-406 (1956).

[4] J. E. TAYLOR, The strongest column-An energy approach. J. appl. Mech. 34, Series E, 486-487 (1967).

[5] J. E. TAYLOR, Minimum mass bar for axial vibration at specified natural frequency. $A I A A J n l 5,1911-1913$ (1967).

[6] E. F. MAsur, On the analysis of buckled plates. Proc. Third U.S. National Congr. Appld. Mech., pp. 411-417 (1958).

[7] K. O. Freidrichs and J. J. Stoker, Buckling of the circular plate beyond the critical thrust. J. appl. Mech. 9, A7-A 14 (1942).

[8] M. R. HeStents, Elements of the calculus of variations, Modern Math. for the Engineer, Chapter 4, p. 82, Edited by E. F. BeCKenBaCF, McGraw-Hill (1956).

(Received 29 December 1969; revised 18 March 1970)

\begin{abstract}
Абсстракт-Конструкции, обладающие некоторым запасом способности после потери устойчивости, могут вообще становиться жестне. В закритической области пугем введения предварительного напряжения. Расчет предварительного напряженного состояния для зтих случаев приводит к максимализации нагрузки вьшучивания для заданной свободной знергии деформации, вытекающеи кз предварительного напряженного состояния. Форммлиравка, представленна для зтого оптимального расчета, тесно связанная с принципами знергии для расчета конструкций. Определяются оптимальные формы для круглой пластинки и для второго примера конструкции типа стержневой мембранной системы. Разработка теории, вытекающая из расчета конструкций с учетом предварительного напряхения, имеет огношение к предыдущим результатам анализа после потери устойчквости.
\end{abstract}

